\title{
Human Equilibrative Nucleoside Transporter 1 Predicts Survival in Patients with Pancreatic Cancer Treated with Gemcitabine: A Meta-Analysis
}

\author{
Yufeng Zhu, Ming Qi, ${ }^{1}$ Lijun Lao, ${ }^{3}$ Wei Wang, Luojie Hua, and Guang Bai ${ }^{1}$
}

\begin{abstract}
Context: Increasing scientific evidence suggests that human equilibrative nucleoside transporter 1 (hENT1) may be a powerful predictor of survival in patients with pancreatic cancer treated with adjuvant gemcitabine-based chemotherapy after operative resection, but many existing studies have yielded inconclusive results. Objective: This meta-analysis aims to assess the prognostic role of hENT1 in predicting survival in patients with pancreatic cancer treated with gemcitabine. Methods: An extensive literature search for relevant studies was conducted on PubMed, Embase, Web of Science, Cochrane Library, and CBM databases from their inception through May 1, 2013. This meta-analysis was performed using the STATA 12.0 software. The crude hazard ratio (HR) with 95\% confidence interval (CI) was calculated. Results: Eleven clinical studies were included in this metaanalysis with a total of 851 pancreatic cancer patients, including 478 patients in the high hENT1 expression group and 373 patients in the low hENT1 expression group. Our meta-analysis revealed that high hENT1 expression was associated with improved overall survival (OS) of pancreatic cancer patients $(\mathrm{HR}=2.61,95 \%$ $\mathrm{CI}=2.02-3.34)$. Pancreatic cancer patients with high hENT1 expression also had a longer disease-free survival (DFS) than those with low hENT1 expression $(\mathrm{HR}=2.62,95 \% \mathrm{CI}=1.94-3.54)$. Further, high hENT1 mRNA showed significant association with improved OS and DFS of pancreatic cancer patients $(\mathrm{HR}=2.65,95 \%$ $\mathrm{CI}=1.75-4.00 ; \mathrm{HR}=3.29,95 \% \mathrm{CI}=1.85-5.84$; respectively). Conclusion: In conclusion, our meta-analysis suggests that high hENT1 expression may be associated with improved OS and DFS of pancreatic cancer patients treated with gemcitabine. Detection of hENT1 expression may be a promising biomarker for gemcitabine response and prognosis in pancreatic cancer patients.
\end{abstract}

\section{Introduction}

$\mathbf{P}$ ANCREATIC CANCER is a highly aggressive cancer and the fourth leading cause of cancer death (Wang et al., 2009). In 2008, an estimated 278,684 new cases and 265,000 deaths as a result of pancreatic cancer occurred worldwide (Siegel et al., 2012). Indeed, even after surgical resection with curative intent, the actuarial 5-year survival rate has been reported to be less than 20\% (Lau et al., 2010). Therefore, surgery alone is not a sufficient treatment for pancreatic cancer, and adjuvant chemoradiation following curative resection has an impact on long-term survival (Traverso, 2006; Hsu et al., 2010). Gemcitabine is a pyrimidine nucleoside analogue used for the treatment of a wide range of solid tumors, including pancreatic cancer (Wonganan et al., 2012). Previous studies have demonstrated that adjuvant chemo- radiation with gemcitabine has a beneficial effect on prognosis in patients with pancreatic cancer after curative resection (Oettle et al., 2007; Van Laethem et al., 2010). Recently, it has been widely accepted that gemcitabine-based regimen is the first-line chemotherapy for patients with unresectable or resected pancreatic cancer (Kanai et al., 2011; Kim et al., 2011b).

The human equilibrative nucleoside transporter 1 (hENT1) protein is the major route for transporting gemcitabine into cells (Garcia-Manteiga et al., 2003; Fujita et al., 2010). It has been recently reported that hENT1 is predictive marker of response to adjuvant chemoradiation with gemcitabine (Garcia-Manteiga et al., 2003; Marechal et al., 2009; Voutsadakis, 2011). In vitro studies also showed that high hENT1 gene expression was significantly associated with increased chemosensitivity to gemcitabine (Mori et al., 2007; Michalski et al., 2008). Several previous studies have indicated

Departments of ${ }^{1}$ Minimally Invasive Surgery, ${ }^{2}$ Ultrasound, and ${ }^{3}$ Hepatobiliary Surgery, The First Affiliated Hospital of Liaoning Medical University, Jinzhou, People's Republic of China. 
that high hENT1 protein/mRNA expression was associated with increased overall survival (OS) and disease-free survival (DFS) (Farrell et al., 2009; Marechal et al., 2012; Morinaga et al., 2012). However, there are also some contradictory data in the literature concerning the prognostic value of hENT1 in predicting gemcitabine chemosensitivity for pancreatic cancer patients (Fujita et al., 2010). The controversial findings are probably related to the effects from interacting with other genes, environmental effects on gene expression, different detection methods, sample sizes, and study design. Therefore, we performed a meta-analysis of all eligible studies to provide insights into the prognostic role of hENT1 in predicting survival in patients with pancreatic cancer treated with gemcitabine.

\section{Materials and Methods}

\section{Search strategy}

An extensive literature search for relevant studies was conducted on PubMed, Embase, Web of Science, Cochrane Library, and CBM databases from their inception through May 1, 2013. We used the following keywords and MeSH terms: ("equilibrative nucleoside transport proteins" or "equilibrative nucleoside transportor" or "ENT" or "hENT" or "SLC29") and ("pancreatic cancer" or "pancreatic neoplasm" or "pancreatic tumor" or "pancreatic carcinoma" or "pancreatic carcinogenesis"), and ("gemcitabine" or "GEM" or "deoxycytidine analog"). There were no language restrictions. Manual search of reference lists from potentially relevant articles was also performed to identify other potential studies.

\section{Selection criteria}

To be included in the meta-analysis, these studies must meet the following criteria: (1) clinical studies focused on the prognostic role of hENT1 in predicting survival in patients with pancreatic cancer treated with gemcitabine; (2) the minimum number of cases in the included studies should be greater than 20; (3) all patients diagnosed with pancreatic cancer were confirmed through histopathologic examinations; (4) published data on DFS and OS estimates were sufficient. Studies were excluded if they did not meet all of the inclusion criteria. If more than one study by the same author using the same case series were published, either the study with the largest sample size or the most recent publication was included. Any disagreements were resolved through discussions and subsequent consensus.

\section{Data extraction}

Two authors independently extracted data from eligible studies using a standardized form. The following information were collected: surname of first author, year of publication, source of publication, country of origin, ethnicity, language of publication, study design, total number of cases, pathological subtype, and detection method of hENT1 expression. In cases of conflicting evaluations, disagreements on inconsistent data from the eligible studies were resolved through discussions and careful reexaminations of the full text by the authors.

\section{Quality assessment}

The quality of the included studies was independently assessed by two authors (C.M.Y. and J.L.) based on the
Newcastle-Ottawa Scale (NOS) criteria for the assessment of the quality of nonrandomized studies (Stang, 2010). The NOS criteria use a "star" rating system to judge the methodological quality, which was based on three perspectives of the study: selection, comparability, and outcome. Scores ranged from 0 stars (worst) to 9 stars (best); a score equal to or greater than 7 indicates a generally good methodological quality. Disagreements on the quality assessment of the included studies were resolved through a comprehensive reassessment by the authors.

\section{Statistical analysis}

Crude hazard ratio (HR) with $95 \%$ confidence interval (CI) was calculated under a fixed or random effect model. The significance of the pooled estimate was determined using the $Z$ test. We estimated the degree of heterogeneity among studies using Cochran's $Q$-statistic, which is considered significant at $p<0.05$ (Jackson et al., 2012). The $I^{2}$ test was also conducted to quantify heterogeneity (ranges from 0 to 100\%) (Biggerstaff and Jackson, 2008). The random-effect model (DerSimonian Laird method) was conducted when there exists a significant $Q$-test with $p<0.05$ or $I^{2}>50 \%$. When there was no statistical heterogeneity, we used the fixed-effects model (Mantel-Haenszel method). To explore potential sources of heterogeneity, subgroup analyses were performed based on ethnicity, treatment regimen, and type of hENT1 expression. To evaluate the influence of single studies on the overall estimate, we conducted a sensitivity analysis by omitting each study in turn to assess the quality and consistency of the results. To investigate whether publication bias might have affected the validity of the estimates, funnel plots were constructed. The symmetry of the funnel plots was further evaluated by Egger's linear regression test (Peters et al., 2006). All tests were two-sided with a $p$-value of $<0.05$ being considered statistically significant. All analyses were calculated using the STATA software, version 12.0 (Stata Corp, College Station, TX).

\section{Results}

\section{Baseline characteristics of included studies}

A total of 57 articles relevant to the searched keywords were initially identified. Of these articles, 23 were excluded after a review of their titles and key words; then, abstracts and full texts were reviewed, and another 23 articles were excluded. Eleven clinical studies met our inclusion criteria for this meta-analysis (Spratlin et al., 2004; Giovannetti et al., 2006; Farrell et al., 2009; Marechal et al., 2009, 2012; Fujita et al., 2010; Kim et al., 2011a; Kondo et al., 2012; Morinaga et al., 2012; Murata et al., 2012; Nakagawa et al., 2013). The flow chart of the study selection process is shown in Figure 1. Publication years of the eligible studies ranged from 2004 to 2013. A total of 851 pancreatic cancer patients were involved in this meta-analysis, including 478 patients in the high hENT1 expression group and 373 patients in the low hENT1 expression group. Overall, six studies were conducted in Caucasian populations, and the other five studies in Asian populations. Detetion methods include immunohistochemistry and quantitative reverse transcription-polymerase chain reaction. The characteristics and methodological quality of the included studies are summarized in Table 1. 
FIG. 1. Flowchart of the literature search and study selection.

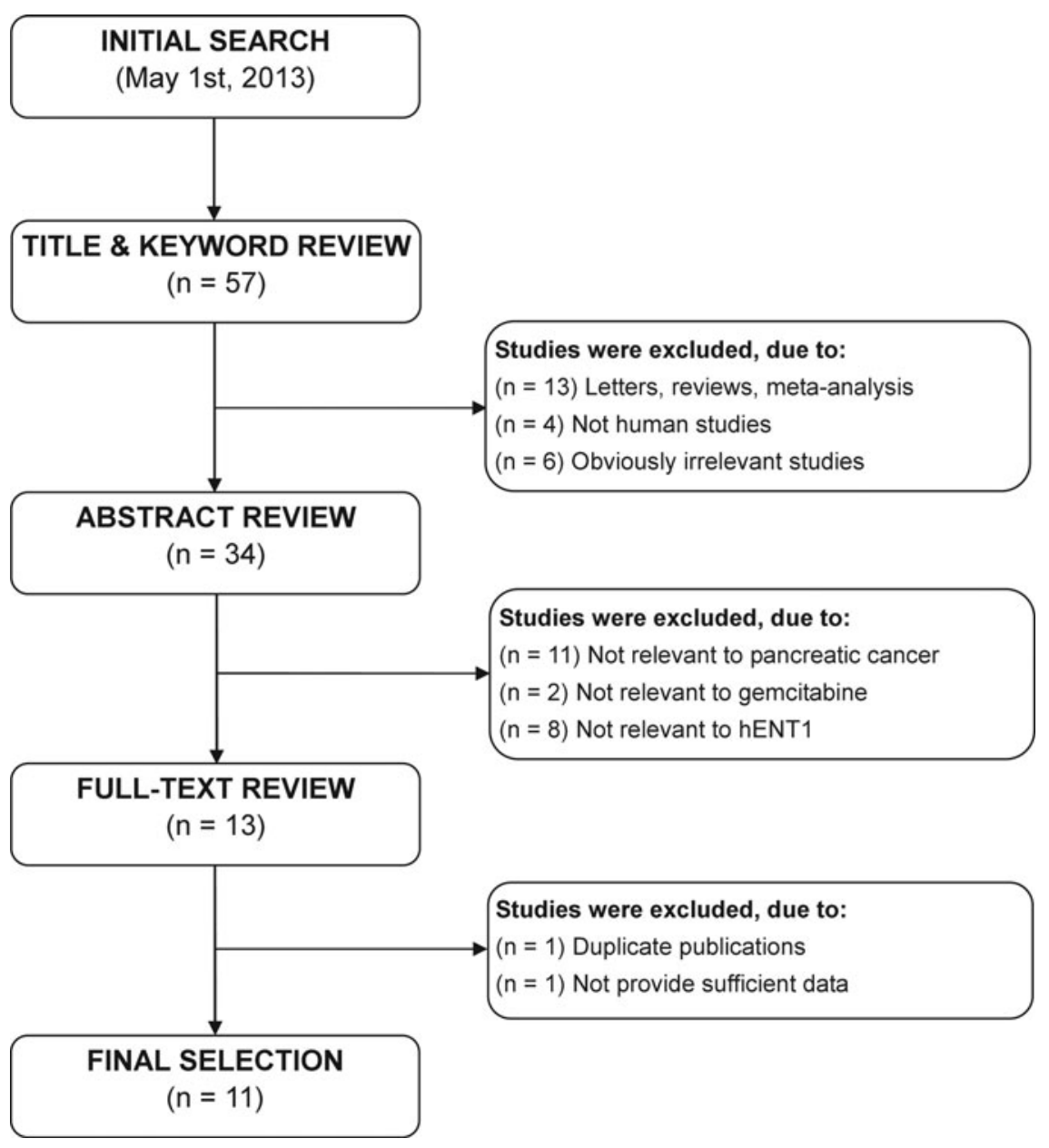

Table 1. Characteristics and Methodological Quality of the Included Studies

\begin{tabular}{|c|c|c|c|c|c|c|c|c|c|c|}
\hline \multirow[b]{2}{*}{$\begin{array}{l}\text { First author } \\
\text { [Ref.] }\end{array}$} & \multirow[b]{2}{*}{ Year } & \multirow[b]{2}{*}{ Country } & \multirow[b]{2}{*}{ Ethnicity } & \multicolumn{2}{|c|}{ Case number } & \multirow[b]{2}{*}{$\begin{array}{l}\text { Treatment } \\
\text { regimen }\end{array}$} & \multirow[b]{2}{*}{$\begin{array}{l}\text { Type of } \\
\text { hENT1 }\end{array}$} & \multirow[b]{2}{*}{ Detection method } & \multirow[b]{2}{*}{ Outcome } & \multirow[b]{2}{*}{ NOS } \\
\hline & & & & $\begin{array}{c}\text { High } \\
\text { hENT1 }\end{array}$ & $\begin{array}{c}\text { Low } \\
\text { hENT1 }\end{array}$ & & & & & \\
\hline Spratlin & 2004 & France & Caucasian & 9 & 12 & GEM alone & Protein & Immunohistochemistry & OS & $5 / 9$ \\
\hline Giovannetti & 2006 & Italy & Caucasian & 37 & 44 & GEM alone & Protein & Immunohistochemistry & OS & $6 / 9$ \\
\hline Farrell & 2009 & $\begin{array}{l}\text { United } \\
\text { States }\end{array}$ & Caucasian & 73 & 18 & $\begin{array}{l}\text { GEM plus } \\
\text { radiotherapy }\end{array}$ & Protein & Immunohistochemistry & OS & $6 / 9$ \\
\hline Marechal & 2009 & Belgium & Caucasian & 19 & 26 & $\begin{array}{l}\text { GEM plus } \\
\text { radiotherapy }\end{array}$ & Protein & Immunohistochemistry & OS/DFS & $7 / 9$ \\
\hline Fujita & 2010 & Japan & Asian & 14 & 26 & $\begin{array}{l}\text { GEM-based } \\
\text { chemotherapy }\end{array}$ & mRNA & qRT-PCR & OS/DFS & $6 / 9$ \\
\hline Kim & 2011 & $\begin{array}{l}\text { United } \\
\text { States }\end{array}$ & Caucasian & 48 & 36 & $\begin{array}{l}\text { GEM-based } \\
\text { chemotherapy }\end{array}$ & mRNA & qRT-PCR & OS/DFS & $7 / 9$ \\
\hline Kondo & 2012 & Japan & Asian & 63 & 23 & $\begin{array}{l}\text { GEM-based } \\
\text { chemotherapy }\end{array}$ & Protein & Immunohistochemistry & OS/DFS & $7 / 9$ \\
\hline Marechal & 2012 & Belgium & Caucasian & 82 & 130 & $\begin{array}{l}\text { GEM-based } \\
\text { chemotherapy }\end{array}$ & Protein & Immunohistochemistry & OS/DFS & $8 / 9$ \\
\hline Morinaga & 2012 & Japan & Asian & 16 & 11 & GEM alone & Protein & Immunohistochemistry & OS/DFS & $7 / 9$ \\
\hline Murata & 2012 & Japan & Asian & 39 & 16 & $\begin{array}{l}\text { GEM plus } \\
\text { radiotherapy }\end{array}$ & Protein & Immunohistochemistry & OS/DFS & $6 / 9$ \\
\hline Nakagawa & 2013 & Japan & Asian & 78 & 31 & $\begin{array}{l}\text { GEM-based } \\
\text { chemotherapy }\end{array}$ & Protein & Immunohistochemistry & OS/DFS & $6 / 9$ \\
\hline
\end{tabular}

hENT1, human equilibrative nucleoside transporter 1; GEM, gemcitabine; qRT-PCR, quantitative reverse transcription-polymerase chain reaction; OS, overall survival; DFS, disease-free survival; NOS, the Newcastle-Ottawa Scale. 
Table 2. Meta-Analysis Findings for the Role of hENT1 Expression in Predicting Survival of Pancreatic Cancer Patients Treated with Gemcitabine

\begin{tabular}{|c|c|c|c|c|c|c|}
\hline \multirow[b]{2}{*}{ Estimates } & \multicolumn{3}{|c|}{ OS } & \multicolumn{3}{|c|}{$D F S$} \\
\hline & $H R$ & $95 \% C I$ & $\mathrm{p}$ & $H R$ & $95 \% C I$ & $\mathrm{p}$ \\
\hline $\begin{array}{l}\text { Overall }(n=11) \\
\text { Ethnicity }\end{array}$ & 2.54 & {$[1.98,3.10]$} & $<0.001$ & 2.71 & {$[1.92,3.50]$} & $<0.001$ \\
\hline Caucasian $(n=6)$ & 2.49 & {$[1.82,3.16]$} & $<0.001$ & 3.18 & {$[1.36,4.99]$} & 0.001 \\
\hline Asian $(n=5)$ & 2.65 & {$[1.63,3.67]$} & $<0.001$ & 2.6 & {$[1.72,3.47]$} & $<0.001$ \\
\hline $\begin{array}{l}\text { Treatment regimen } \\
\text { GEM alone }(n=3)\end{array}$ & 3.23 & {$[1.32,5.13]$} & $<0$ & 2.56 & $55]$ & $<0.001$ \\
\hline GEM plus radiotherapy $(n=3)$ & 2.76 & {$[1.49,4.03]$} & $<0.001$ & 2.78 & {$[1.76,3.79]$} & $<0.001$ \\
\hline $\begin{array}{l}\text { GEM-based chemotherapy }(n=5) \\
\text { Type of hENT1 }\end{array}$ & 2.39 & {$[1.73,3.05]$} & $<0.001$ & 2.76 & {$[0.11,5.63]$} & 0.049 \\
\hline Protein $(n=9)$ & 2.63 & {$[1.99,3.27]$} & $<0.001$ & 2.61 & {$[1.76,3.47]$} & $<0.001$ \\
\hline mRNA $(n=2)$ & 2.25 & {$[1.12,3.38]$} & $<0.001$ & 3.27 & {$[1.18,5.36]$} & 0.002 \\
\hline
\end{tabular}

HR, hazard ratio; 95\% CI, 95\% confidence interval.

\section{Quantitative data synthesis}

A summary of the meta-analysis findings for the role of the expression of hENT1 in predicting survival of pancreatic cancer patients treated with gemcitabine is provided in Table 2 . Since heterogeneity obviously existed, which could be a result of differences in ethnicity, treatment regimen, and type of hENT1 expression, the random effects model was conducted. The meta-analysis results revealed that high hENT1 expression was associated with improved OS of pancreatic cancer patients $(\mathrm{HR}=2.54,95 \% \mathrm{CI}=1.98-3.10, p<0.001)$ (Fig. 2). Pancreatic cancer patients with high hENT1 expression also had a longer DFS than those with low hENT1 expression $(\mathrm{HR}=2.71,95 \% \mathrm{CI}=1.92-3.50, p<0.001)$ (Fig. 3).
Subgroup analysis by ethnicity indicated that high hENT1 expression may improve OS and DFS of pancreatic cancer patients among Caucasian and Asian populations (all $p<0.05)$. Further subgroup analysis by treatment regimen and type of hENT1 expression also showed significant associations between high hENT1 expression and improved OS and DFS of pancreatic cancer patients in each subgroup (as shown in Table 2).

\section{Evaluation of heterogeneity and publication bias}

Sensitivity analysis was also performed to assess the influence of each individual study on the pooled HR by omitting each individual studies in turn to assess the quality and consistency of

\begin{tabular}{|c|c|c|c|}
\hline Study ID & $\begin{array}{c}\text { Overall survival } \\
\text { (High hENT1 versus Low hENT1) }\end{array}$ & $\mathrm{HR}(95 \% \mathrm{Cl})$ & Weight $\%$ \\
\hline Spratlin JR (2004) & & $3.25(1.33,7.97)$ & 2.84 \\
\hline Giovannetti EM (2006) & & $4.21(1.79,9.91)$ & 1.90 \\
\hline Farrell JJ (2009) & & $2.50(1.35,4.62)$ & 11.70 \\
\hline Marechal R (2009) & & $3.42(1.38,8.46)$ & 2.50 \\
\hline Fujita H (2010) & & $2.98(0.89,10.00)$ & 1.51 \\
\hline Kim R (2011) & & $2.20(1.32,3.66)$ & 22.85 \\
\hline Kondo N (2012) & & $2.18(1.10,4.19)$ & 13.10 \\
\hline Marechal R (2012) & & $2.44(1.59,3.70)$ & 28.10 \\
\hline Morinaga S (2012) & & $2.73(1.10,6.76)$ & 3.91 \\
\hline Murata Y (2012) & & $3.04(1.45,6.37)$ & 5.17 \\
\hline Nakagawa N (2012) & & $3.16(1.65,6.06)$ & 6.43 \\
\hline Overall $\left(I^{2}=53.0 \%, P=0.045\right)$ & & $2.54(1.98,3.10)$ & 100.00 \\
\hline NOTE: Random effects analysis & & & \\
\hline $\begin{array}{l}T \\
-10\end{array}$ & 0 & & \\
\hline
\end{tabular}

FIG. 2. Forest plots for the associations between the human equilibrative nucleoside transporter 1 (hENT1) expression and overall survival (OS) in pancreatic cancer patients who received gemcitabine. The squares and horizontal lines correspond to the study specific hazard ratio (HR) and $95 \%$ confidence interval $(95 \% \mathrm{CI})$. The area of the squares reflects the weight. The diamond represents the summary HR and 95\% CI. 


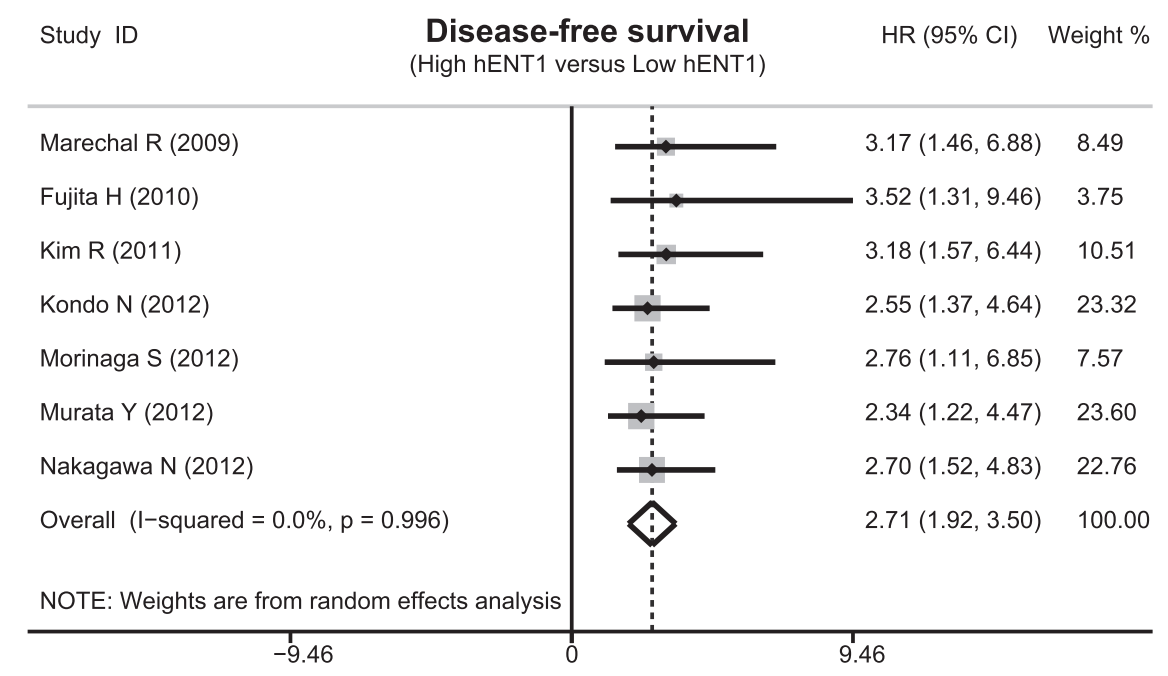

FIG. 3. Forest plots for the associations between the hENT1 expression and disease-free survival (DFS) in pancreatic cancer patients who received gemcitabine. The squares and horizontal lines correspond to the study-specific HR and 95\% CI. The area of the squares reflects the weight. The diamond represents the summary HR and $95 \% \mathrm{CI}$.
Egger's test also did not display strong statistical evidence for publication bias (all $p>0.05$ ). studies significantly affected the pooled HRs. Funnel plots and Egger's linear regression test were used to assess potential publication bias in the included studies. The shapes of the funnel plots did not reveal any evidence of obvious asymmetry (Fig. 4).
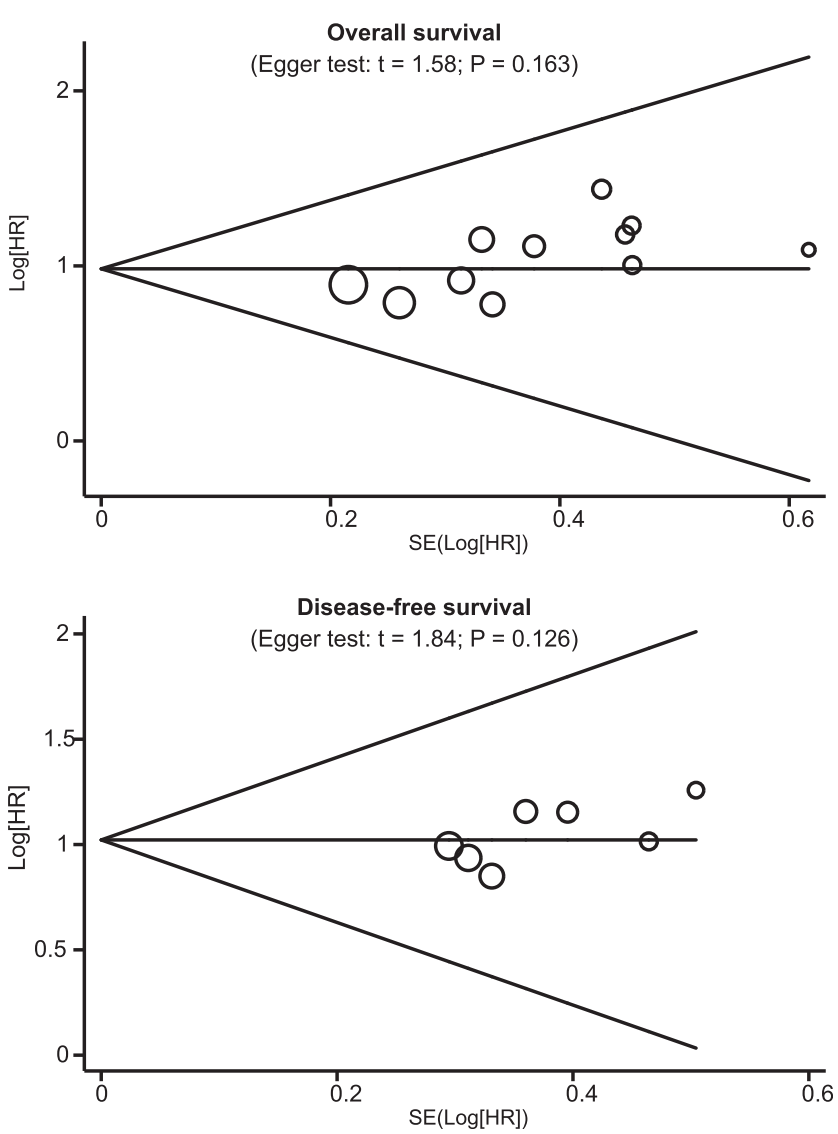

FIG. 4. Funnel plots of the meta-analysis show the pooled HR estimates of the prognostic role of the hENT1 expression in predicting survival of pancreatic cancer patients who received gemcitabine. Each point represents a separate study for the indicated association. $\log [\mathrm{HR}]$, natural logarithm of HR. Horizontal line, mean magnitude of the effect. Note: Funnel plot with pseudo 95\% confidence limits was used.

\section{Discussion}

Pancreatic cancer is one of the most lethal human cancers with an overall 5-year survival rate of less than 5\% (Li et al., 2004; Neoptolemos et al., 2010). However, during the last decade, few successes have been archived in the treatment of pancreatic cancer (Choi et al., 2012).The most successful agent for pancreatic cancer treatment is gemcitabine, although the overall effect in terms of patient survival remains very poor (Kovacevic et al., 2011). Cellular uptake of the anticancer drug gemcitabine is mainly mediated by hENT1 (Robins et al., 2010). It has been hypothesized that deficiency of the hENT1 transporter may contribute to clinical gemcitabine resistance (Lai et al., 2008). Increasingly strong evidence suggests hENT1 is a prognostic biomarker in gemcitabine-treated pancreatic cancer, and may well be a predictive biomarker of gemcitabine efficacy (Spratlin et al., 2004; Murata et al., 2012). There is a compelling biological rationale for using the expression level of hENT1 to predict gemcitabine chemosensitivity and prognosis of pancreatic cancer patients.

Our meta-analysis suggested that high hENT1 expression was associated with improved OS and DFS of pancreatic cancer patients treated with gemcitabine-based regimens. These results indicated that the efficacy of adjuvant chemoradiation with gemcitabine is associated with the expression level of hENT1, which is consistent with previous studies. Farrell et al. (2009) reported that hENT1 protein expression was associated with increased DFS and OS in pancreatic cancer patients who received gemcitabine, but not in those who received 5-fluorouracil (5-FU). Murata et al. (2012) demonstrated that the 1- and 3-year OS rates were significantly greater in the high hENT1 expression group than in the low hENT1 expression group. Other previous studies also indicated that the hENT1 expression was a significant and independent prognostic factor for OS (Marechal et al., 2012; Morinaga et al., 2012) In the current study, we also performed subgroup analyses based on ethnicity, treatment regimen, and type of hENT1 expression to further evaluate the prognostic value of hENT1 expression for survival in 
pancreatic cancer patients who received gemcitabine. The results strongly suggested that detection of hENT1 expression may be a promising biomarker for gemcitabine response and prognosis in pancreatic cancer patients.

Indeed, other than gemcitabine-based regimens, there do exist several other methods that have significant anticancer effects on pancreatic cancer (Conroy et al., 2011; Seufferlein, 2013). However, without knowledge of genetic background of certain disease, chemotherapy administered to patients may affect drug efficacy (Borbath et al., 2012). For example, 5-FU, S-1 (TS-1), capecitabine, irinotecan, and 6-mercaptopurine are current therapeutic options (Giovannetti et al., 2006; Tempero et al., 2011). Among them, 5-FU, previous experiment results had shown that 5-FU might lead to an increase in cell surface hENT1, which could potentially augment drug efficacy (Nakata et al., 2010). Although concurrent treatment or gemcitabine prior to 5-FU rarely existed, pancreatic cancer cell pretreatment lines in vitro and in vivo with 5-FU were usually adopted to strengthen the effects of single-agent gemcitabine treatment (Nakahira et al., 2008). In addition, treatment with $\mathrm{S}-1$ resulted in hENT1 upregulation at the mRNA level, and thus S-1 was more convenient and helps improvement of median survival time in pancreatic cancer (Ueno et al., 2011; Song et al., 2013). Another drug, capecitabine, had shown activity in combination with gemcitabine in locally advanced or metastatic pancreatic cancer patients with regard to the assessment of hENT1 (Pliarchopoulou and Pectasides, 2009). Nevertheless, evidence for other biomarkers is limited by a small number of publications investigating these markers; hence, we do not present a further meta-analysis on the above treatment options.

Our meta-analysis has several limitations that should be acknowledged. The first major limitation is the relatively small sample size of this meta-analysis, which may not have sufficient statistical power in estimating the prognostic role of hENT1 expression in pancreatic cancer. Therefore, more studies with larger sample size are still needed. On the other hand, as a type of a retrospective study, a meta-analysis of summary data from previously published studies may encounter recall or selection bias, thereby possibly influencing the reliability of the results. Most important of all, the lack of access to all the data from the original studies limited further evaluations of the potential values of hENT1 expression. However, despite these statistical limitations, our study is the first comprehensive meta-analysis of all eligible studies concerning the prognostic role of hENT1 expression in predicting survival in patients with pancreatic cancer treated with gemcitabine.

In conclusion, our meta-analysis indicates that high hENT1 expression may be associated with improved OS and DFS of pancreatic cancer patients treated with gemcitabine. Detection of hENT1 expression may be a promising biomarker for gemcitabine response and prognosis in pancreatic cancer patients. However, due to the limitations mentioned above, further detailed studies are still required to confirm our findings.

\section{Acknowledgments}

We would like to acknowledge the helpful comments on this article received from reviewers. We thank all our colleagues working in the Department of Minimally Invasive
Surgery, The First Affiliated Hospital of Liaoning Medical University. The work was funded by the Youth Science and Technology Foundation of The First Affiliated Hospital of Liaoning Medical University (FY2012-2013).

\section{Author Disclosure Statement}

No competing financial interests exist.

\section{References}

Biggerstaff BJ, Jackson D (2008) The exact distribution of Cochran's heterogeneity statistic in one-way random effects meta-analysis. Stat Med 27:6093-6110.

Borbath I, Verbrugghe L, Lai R, et al. (2012) Human equilibrative nucleoside transporter 1 (hENT1) expression is a potential predictive tool for response to gemcitabine in patients with advanced cholangiocarcinoma. Eur J Cancer 48: 990-996.

Choi M, Kim R, Saif MW (2012) What options are available for refractory pancreatic cancer? JOP 13:163-165.

Conroy T, Desseigne F, Ychou M, et al. (2011) FOLFIRINOX versus gemcitabine for metastatic pancreatic cancer. N Engl J Med 364:1817-1825.

Farrell JJ, Elsaleh H, Garcia M, et al. (2009) Human equilibrative nucleoside transporter 1 levels predict response to gemcitabine in patients with pancreatic cancer. Gastroenterology 136:187-195.

Fujita H, Ohuchida K, Mizumoto K, et al. (2010) Gene expression levels as predictive markers of outcome in pancreatic cancer after gemcitabine-based adjuvant chemotherapy. Neoplasia 12:807-817.

Garcia-Manteiga J, Molina-Arcas M, Casado FJ, et al. (2003) Nucleoside transporter profiles in human pancreatic cancer cells: role of hCNT1 in $2^{\prime}, 2^{\prime}$-difluorodeoxycytidine- induced cytotoxicity. Clin Cancer Res 9:5000-5008.

Giovannetti E, Del Tacca M, Mey V, et al. (2006) Transcription analysis of human equilibrative nucleoside transporter-1 predicts survival in pancreas cancer patients treated with gemcitabine. Cancer Res 66:3928-3935.

Hsu CC, Herman JM, Corsini MM, et al. (2010) Adjuvant chemoradiation for pancreatic adenocarcinoma: the Johns Hopkins Hospital-Mayo Clinic collaborative study. Ann Surg Oncol 17:981-990.

Jackson D, White IR, Riley RD (2012) Quantifying the impact of between-study heterogeneity in multivariate meta-analyses. Stat Med 31:3805-3820.

Kanai M, Yoshimura K, Asada M, et al. (2011) A phase I/II study of gemcitabine-based chemotherapy plus curcumin for patients with gemcitabine-resistant pancreatic cancer. Cancer Chemother Pharmacol 68:157-164.

Kim R, Tan A, Lai KK, et al. (2011a) Prognostic roles of human equilibrative transporter 1 (hENT-1) and ribonucleoside reductase subunit M1 (RRM1) in resected pancreatic cancer. Cancer 117:3126-3134.

Kim ST, Lim do H, Jang KT, et al. (2011b) Impact of KRAS mutations on clinical outcomes in pancreatic cancer patients treated with first-line gemcitabine-based chemotherapy. Mol Cancer Ther 10:1993-1999.

Kondo N, Murakami Y, Uemura K, et al. (2012) Combined analysis of dihydropyrimidine dehydrogenase and human equilibrative nucleoside transporter 1 expression predicts survival of pancreatic carcinoma patients treated with adjuvant gemcitabine plus S-1 chemotherapy after surgical resection. Ann Surg Oncol 19 Suppl 3:S646-S655. 
Kovacevic Z, Chikhani S, Lovejoy DB, et al. (2011) Novel thiosemicarbazone iron chelators induce up-regulation and phosphorylation of the metastasis suppressor N-myc down-stream regulated gene 1: a new strategy for the treatment of pancreatic cancer. Mol Pharmacol 80:598-609.

Lai R, Bartlett NL, Mackey JR, et al. (2008) High expression of nucleoside transporter protein hENT1 in Reed-Sternberg cells is associated with treatment failure in relapsed/refractory Hodgkin lymphoma patients treated with gemcitabine, vinorelbine and liposomal doxorubicin-a CALGB 59804 correlative study. Leuk Lymphoma 49:1202-1205.

Lau MK, Davila JA, Shaib YH (2010) Incidence and survival of pancreatic head and body and tail cancers: a population-based study in the United States. Pancreas 39:458-462.

Li D, Xie K, Wolff R, et al. (2004) Pancreatic cancer. Lancet 363:1049-1057.

Marechal R, Bachet JB, Mackey JR, et al. (2012) Levels of gemcitabine transport and metabolism proteins predict survival times of patients treated with gemcitabine for pancreatic adenocarcinoma. Gastroenterology 143:664-674; e661-e666.

Marechal R, Mackey JR, Lai R, et al. (2009) Human equilibrative nucleoside transporter 1 and human concentrative nucleoside transporter 3 predict survival after adjuvant gemcitabine therapy in resected pancreatic adenocarcinoma. Clin Cancer Res 15:2913-2919.

Michalski CW, Erkan M, Sauliunaite D, et al. (2008) Ex vivo chemosensitivity testing and gene expression profiling predict response towards adjuvant gemcitabine treatment in pancreatic cancer. Br J Cancer 99:760-767.

Mori R, Ishikawa T, Ichikawa Y, et al. (2007) Human equilibrative nucleoside transporter 1 is associated with the chemosensitivity of gemcitabine in human pancreatic adenocarcinoma and biliary tract carcinoma cells. Oncol Rep 17:1201-1205.

Morinaga S, Nakamura Y, Watanabe T, et al. (2012) Immunohistochemical analysis of human equilibrative nucleoside transporter-1 (hENT1) predicts survival in resected pancreatic cancer patients treated with adjuvant gemcitabine monotherapy. Ann Surg Oncol 19 Suppl 3:S558-S564.

Murata Y, Hamada T, Kishiwada M, et al. (2012) Human equilibrative nucleoside transporter 1 expression is a strong independent prognostic factor in UICC T3-T4 pancreatic cancer patients treated with preoperative gemcitabinebased chemoradiotherapy. J Hepatobiliary Pancreat Sci 19: 413-425.

Nakagawa N, Murakami Y, Uemura K, et al. (2013) Combined analysis of intratumoral human equilibrative nucleoside transporter 1 (hENT1) and ribonucleotide reductase regulatory subunit M1 (RRM1) expression is a powerful predictor of survival in patients with pancreatic carcinoma treated with adjuvant gemcitabine-based chemotherapy after operative resection. Surgery 153:565-575.

Nakahira S, Nakamori S, Tsujie M, et al. (2008) Pretreatment with S-1, an oral derivative of 5-fluorouracil, enhances gemcitabine effects in pancreatic cancer xenografts. Anticancer Res 28:179-186.

Nakata B, Amano R, Nakao S, et al. (2010) Plasma pharmacokinetics after combined therapy of gemcitabine and oral S-1 for unresectable pancreatic cancer. J Exp Clin Cancer Res 29:15.

Neoptolemos JP, Stocken DD, Bassi C, et al. (2010) Adjuvant chemotherapy with fluorouracil plus folinic acid vs gemcitabine following pancreatic cancer resection: a randomized controlled trial. JAMA 304:1073-1081.

Oettle H, Post S, Neuhaus P, et al. (2007) Adjuvant chemotherapy with gemcitabine vs observation in patients under- going curative-intent resection of pancreatic cancer: a randomized controlled trial. JAMA 297:267-277.

Peters JL, Sutton AJ, Jones DR, et al. (2006) Comparison of two methods to detect publication bias in meta-analysis. JAMA 295:676-680.

Pliarchopoulou K, Pectasides D (2009) Pancreatic cancer: current and future treatment strategies. Cancer Treat Rev 35:431-436.

Robins MJ, Peng Y, Damaraju VL, et al. (2010) Improved syntheses of 5'-S-(2-aminoethyl)-6-N-(4-nitrobenzyl)-5'thioadenosine (SAENTA), analogues, and fluorescent probe conjugates: analysis of cell-surface human equilibrative nucleoside transporter 1 (hENT1) levels for prediction of the antitumor efficacy of gemcitabine. J Med Chem 53:60406053.

Seufferlein T (2013) Systemic treatment of advanced pancreatic cancer-step by step progress. Gut 62:660-661.

Siegel R, Naishadham D, Jemal A (2012) Cancer statistics, 2012. CA Cancer J Clin 62:10-29.

Song H, Han B, Park CK, et al. (2013) Phase II trial of gemcitabine and S-1 for patients with advanced pancreatic cancer. Cancer Chemother Pharmacol 72:845-852.

Spratlin J, Sangha R, Glubrecht D, et al. (2004) The absence of human equilibrative nucleoside transporter 1 is associated with reduced survival in patients with gemcitabine-treated pancreas adenocarcinoma. Clin Cancer Res 10:6956-6961.

Stang A (2010) Critical evaluation of the Newcastle-Ottawa scale for the assessment of the quality of nonrandomized studies in meta-analyses. Eur J Epidemiol 25:603-605.

Tempero MA, Berlin J, Ducreux M, et al. (2011) Pancreatic cancer treatment and research: an international expert panel discussion. Ann Oncol 22:1500-1506.

Traverso LW (2006) Pancreatic cancer: surgery alone is not sufficient. Surg Endosc 20 Suppl 2:S446-S449.

Ueno H, Okusaka T, Furuse J, et al. (2011) Multicenter phase II study of gemcitabine and S-1 combination therapy (GS Therapy) in patients with metastatic pancreatic cancer. Jpn J Clin Oncol 41:953-958.

Van Laethem JL, Hammel P, Mornex F, et al. (2010) Adjuvant gemcitabine alone versus gemcitabine-based chemoradiotherapy after curative resection for pancreatic cancer: a randomized EORTC-40013-22012/FFCD-9203/GERCOR phase II study. J Clin Oncol 28:4450-4456.

Voutsadakis IA (2011) Molecular predictors of gemcitabine response in pancreatic cancer. World J Gastrointest Oncol 3:153-164.

Wang Z, Li Y, Kong D, et al. (2009) Acquisition of epithelialmesenchymal transition phenotype of gemcitabine-resistant pancreatic cancer cells is linked with activation of the notch signaling pathway. Cancer Res 69:2400-2407.

Wonganan P, Chung WG, Zhu S, et al. (2012) Silencing of ribonucleotide reductase subunit M1 potentiates the antitumor activity of gemcitabine in resistant cancer cells. Cancer Biol Ther 13:908-914.

Address correspondence to: Guang Bai, MD

Department of Minimally Invasive Surgery The First Affiliated Hospital of Liaoning Medical University Renmin Street No. 2

Guta District

Jinzhou 121001

People's Republic of China

E-mail:1mu1h_bg@163.com 